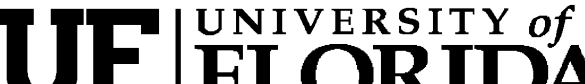 FLORIDA \\ IFAS Extension
}

\section{Dairy Business Analysis Project: 2002 Financial Summary 1}

\author{
A. de Vries, R. Giesy, L. Ely, A. de Araujo, A. Andreasen, B. Broaddus, S. Eubanks, D. Mayo, P. \\ Miller, T. Seawright, C. Vann ${ }^{2}$
}

\section{Introduction}

The Dairy Business Analysis Project (DBAP) was initiated in 1996 by the University of Florida in an effort to measure and document the financial performance of Florida dairy businesses using standardized accounting measures. The University of Georgia has been a formal collaborator since 1998. A committee of dairy farmers appointed by Southeast Milk, Inc. oversees the project and helps direct its course. The DBAP website is http://dairy.ifas.ufl.edu/programs/dbap.shtml

Financial data for the year 2002 were collected from participating dairies and screened for completeness and validity. Each dairy then received a report detailing its financial results compared to the average results for the other participants and the six dairies with the highest net farm income from operations per cwt.

This publication is a summary of the financial performance in the year 2002. It is intended for general use by dairy farmers, the allied industry, and government, and educational professionals.

\section{Data Collection and Accounting Methods}

Dairy businesses in Florida, Georgia, and Alabama were asked to participate in DBAP. Participants were therefore not a random sample of all dairies in the three states.

Most of the data were collected by extension agents when visiting farms using a standardized data collection spreadsheet. Occasionally, data were sent in by the dairy farms. The financial data were either entered into the spreadsheet on the farm or mailed in on paper copies of the spreadsheet.

The accounting methods follow the recommendations made by the Farm Financial Standards Council ${ }^{3}$. All revenues and expenses were accrual adjusted. Cash receipts and expenses were therefore adjusted for changes in inventory, prepaid expenses, depreciation, accounts payable, and

1. This document is AN149, one of a series of the Department of Animal Sciences, Florida Cooperative Extension Service, UF/IFAS. Published June 2004. Please visit the EDIS Web Site at http://edis.ifas.ufl.edu.

2. A. de Vries, Assistant Professor, Department of Animal Sciences; R. Giesy, Extension Agent III; L. Ely, Professor, Department of Animal and Dairy Science, University of Georgia; A. de Araujo, Graduate Student; A. Andreasen, Extension Agent IV; B. Broaddus, Extension Agent I; S. Eubanks, Extension Agent III; D. Mayo, Extension Agent II; P. Miller, Extension Agent IV; T. Seawright, Extension Agent IV; C. Vann, Extension Agent II;. Cooperative Extension Service, UF/IFAS, Gainesville, 32611.

The Institute of Food and Agricultural Sciences (IFAS) is an Equal Opportunity Institution authorized to provide research, educational information and other services only to individuals and institutions that function with non-discrimination with respect to race, creed, color, religion, age, disability, sex, sexual orientation, marital status, national origin, political opinions or affiliations. U.S. Department of Agriculture, Cooperative Extension Service, University of Florida, IFAS, Florida A. \& M. University Cooperative Extension Program, and Boards of County Commissioners Cooperating. Larry Arrington, Dean 
receivable. Depreciation data were taken from tax records. Asset valuation was based on market values, but the changes from January 1 to December 31 were kept small. Unpaid management was valued at $\$ 50,000$ per farm. Gain/loss on sale of capital livestock resulted when livestock depreciation did not completely account for the gain or loss in the value of the purchased livestock during 2002. Appreciation resulted when machinery and building depreciation did not completely account for the gain or loss in the value of these capital assets during 2002.

The bottom line of each dairy business is its net farm income. Net farm income is the return to the owner and unpaid family members for their labor, management, and equity in the business. It is the total income available for owner's salary, new investments, taxes, and paying off principal.

All submitted data were carefully scrutinized and checked for completeness. The cash flow statement reconciles the net cash flow resulting from the reported operating, investing, and financing activities with the reported available cash on the January 1 and December 31 balance sheets. The equity statement reconciles the changes in equity through reported retained capital and valuation with the calculated equity on the balance sheets. The reconciliation attempts typically result in unresolved imbalances. Both cash flow imbalance and equity imbalance had to be less than $10 \%$ for the business to be included in the summary results reported here.

\section{Results}

Twenty-nine dairies submitted financial data. Twenty-seven dairies were included in the summary results. Of these, 18 were located in Florida, 8 in Georgia, and one in Alabama. The average herd size of the participating dairies was 1,168 cows and 583 heifers with $16,810 \mathrm{lbs}$. milk sold per cow. The average culling rate was $34 \%$. The milk price received by DBAP participants averaged $\$ 16.05,12 \%$ less than in 2001. Average total revenues were $\$ 17.67$ per cwt. sold, a decrease of \$2.33 per cwt. compared to 2001.

Total expenses averaged $\$ 17.88$ per cwt., an increase of $\$ 0.13$ compared to 2001. The largest items were purchased feed (\$7.00) and personnel costs (\$2.88). Net farm income from operations averaged
$\$-0.21$ per cwt., a decrease of $\$ 2.46$ compared to 2001. Net farm income per cwt. was \$-0.10.

Summary results are presented in Figures 1 through 5 and Tables 1.1 through 4.4. Some definitions and calculation rules are as follows:

- Asset turnover ratio = total revenues / average assets

- Assets = value of assets on the balance sheet

- Average assets = average of value of assets on January 1 and December 31

- Average equity = average of value of equity on January 1 and December 31

- Capital replacement and term debt repayment margin $=$ net farm income from operations (NFIFO) + depreciation + interest on term debt - net social security and income taxes - owner withdrawals annual scheduled payments on term debt and capital leases

- Cash flow coverage ratio $=($ cash revenues cash expenses) / current liabilities

- Current assets = short-term assets that can be utilized within one year

- Current liabilities = liabilities due within one year

- Current ratio $=$ current assets $/$ current liabilities

- Debt to asset ratio $=$ liabilities $/$ assets

- Debt to equity ratio = liabilities / equity

- Depreciation expense ratio $=$ depreciation $/$ total revenue

- Equity = assets - liabilities

- Equity to asset ratio = equity / assets

- FTE = full time equivalent worker, on average 54 hours per week

- Interest expense ratio $=$ interest paid $/$ total revenue 
- Liabilities = value of liabilities on the balance sheet

- Net farm income $=$ NFIFO + gain on sale of capital assets

- $\mathrm{NFIFO}=$ net farm income from operations

- NFIFO ratio $=$ NFIFO $/$ total revenue

- Operating expense ratio $=($ total operating expenses - depreciation) / total revenue

- Operating profit margin ratio $=(\mathrm{NFIFO}+$ interest paid - unpaid management) / total revenues

- Rate of return on dairy assets $=(\mathrm{NFIFO}+$ interest paid - unpaid management) / average assets

- Rate of return on equity $=(\mathrm{NFIFO}-$ unpaid management) / average equity

- Term debt and capital lease coverage ratio $=$ (NFIFO + non dairy income + depreciation + interest paid on term debt - net social security and income taxes - owner withdrawals) / (annual scheduled payments on term debt and capital leases)

- Working capital $=$ current assets $/$ current liabilities

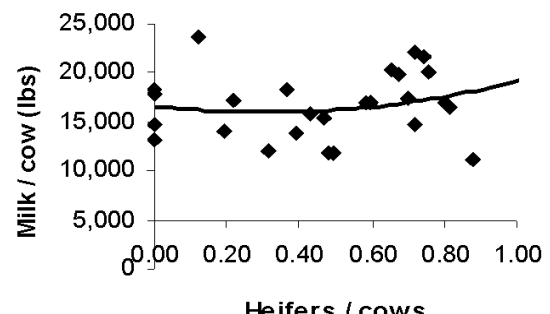

Figure 1. DBAP 2002 Summary - Milk per cow (lbs/year) by heifers per cows.

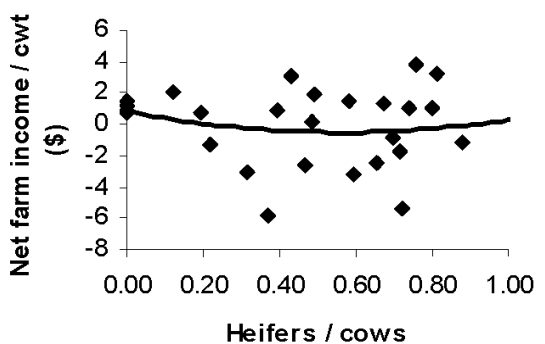

Figure 2. DBAP 2002 Summary - Net farm income per cwt (\$) by heifers per cows.

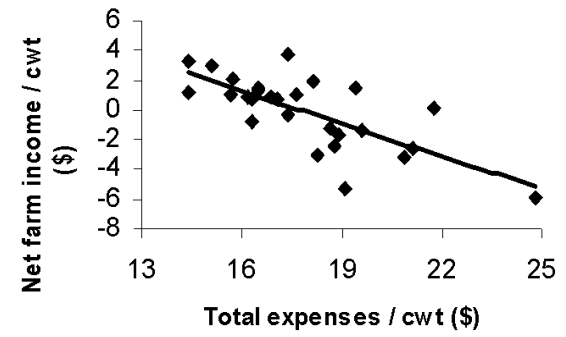

Figure 3. DBAP 2002 Summary - Net farm income per cwt (\$) by total expenses per cwt (\$).

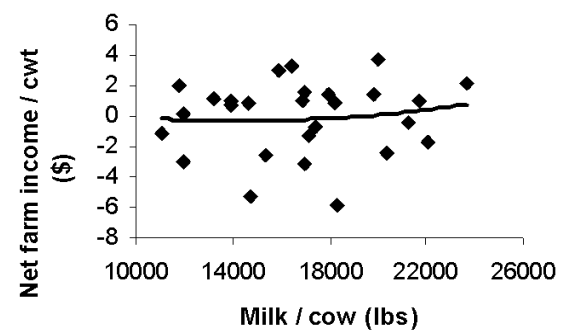

Figure 4. DBAP 2002 Summary - Net farm income per cwt (\$) by milk per cow (lbs/year).

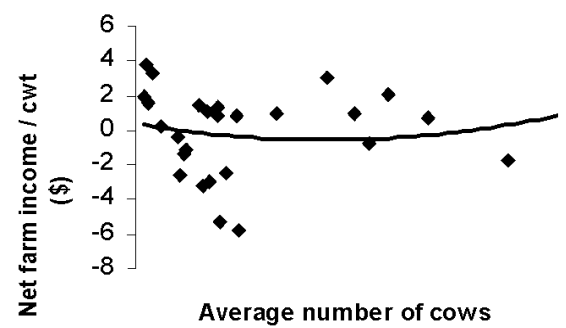

Figure 5. DBAP 2002 Summary - Net farm income per cwt (\$) by average number of cows.

\section{Additional Notes:}

3. Farm Financial Standards Council. 1997. Financial Guidelines for Agricultural Producers. 
Table 1.1. DBAP 2002 Summary - Business size and production efficiency by state and overall average, median, and standard deviation.

\begin{tabular}{|c|c|c|c|c|c|}
\hline \multirow[b]{2}{*}{ Category } & \multicolumn{3}{|c|}{ Overall } & \multicolumn{2}{|c|}{ State Averages } \\
\hline & Average & Median & $\mathrm{Std}^{1}$ & Florida & Georgia \\
\hline Number of farms & 27 & 27 & 27 & 18 & 8 \\
\hline \multicolumn{6}{|l|}{ Business size } \\
\hline Average number of cows & 1,168 & 800 & 1,130 & 940 & 1,297 \\
\hline Average number of heifers & 583 & 366 & 814 & 339 & 724 \\
\hline Milk sold (million lbs) & 21.23 & 11.71 & 24.03 & 18.66 & 23.04 \\
\hline FTE workers & 20 & 12 & 22 & 15 & 23 \\
\hline Acres of pasture + cultivated land & 606 & 358 & 584 & 400 & 717 \\
\hline \multicolumn{6}{|l|}{ Production efficiency } \\
\hline Milk sold/cow (lbs) & 16,810 & 16,940 & 3,418 & 16,118 & 18,812 \\
\hline Cows/FTE worker & 61 & 59 & 24 & 54 & 63 \\
\hline Milk sold/FTE worker (million lbs) & 1.01 & 0.90 & 0.43 & 1.04 & 1.00 \\
\hline Cull rate & 0.34 & .35 & 0.11 & 0.35 & 0.34 \\
\hline
\end{tabular}

${ }^{1}$ Standard deviation.

Table 1.2. DBAP 2002 Summary - Revenues and expenses by state and overall average, median, and standard deviation.

\begin{tabular}{|c|c|c|c|c|c|}
\hline \multirow[b]{2}{*}{ Category } & \multicolumn{3}{|c|}{ Overall } & \multicolumn{2}{|c|}{ State Averages } \\
\hline & Average & Median & $\mathrm{Std}^{1}$ & Florida & Georgia \\
\hline Number of farms & 27 & 27 & 27 & 18 & 8 \\
\hline \multicolumn{6}{|l|}{ Revenues (per cwt) } \\
\hline Milk sold (\$) & 16.05 & 16.07 & 1.01 & 16.12 & 16.15 \\
\hline Raised, leased cow sales (\$) & 0.65 & 0.60 & 0.74 & 0.87 & 0.59 \\
\hline Heifer sales $(\$)$ & 0.13 & 0.13 & 0.65 & 0.02 & 0.17 \\
\hline Gain on purchased livestock sales (\$) & $(0.32)$ & 0.00 & 1.44 & $(0.47)$ & $(0.28)$ \\
\hline Other revenues $(\$)$ & 1.16 & 0.71 & 1.71 & 1.18 & 1.13 \\
\hline Total revenues (\$) & 17.67 & 17.68 & 1.94 & 17.72 & 17.77 \\
\hline \multicolumn{6}{|l|}{ Expenses (per cwt) } \\
\hline Personnel (\$) & 2.88 & 2.91 & 0.91 & 2.20 & 3.26 \\
\hline Purchased feed $(\$)$ & 7.00 & 6.36 & 1.89 & 5.41 & 7.84 \\
\hline Crops $(\$)$ & 0.34 & 0.21 & 0.43 & 0.62 & 0.22 \\
\hline Machinery (\$) & 0.81 & 0.78 & 0.44 & 0.79 & 0.83 \\
\hline Livestock (\$) & 1.84 & 1.73 & 0.91 & 2.10 & 1.76 \\
\hline Milk marketing (\$) & 1.08 & 0.93 & 0.40 & 1.56 & 0.90 \\
\hline Buildings and land (\$) & 0.69 & 0.45 & 0.72 & 1.06 & 0.56 \\
\hline Interest (\$) & 0.51 & 0.39 & 0.43 & 0.34 & 0.54 \\
\hline \multicolumn{6}{|l|}{ Depreciation: } \\
\hline Livestock (\$) & 1.04 & 0.84 & 0.98 & 0.78 & 1.02 \\
\hline Machinery (\$) & 0.55 & 0.44 & 0.46 & 0.75 & 0.49 \\
\hline Buildings (\$) & 0.30 & 0.22 & 0.33 & 0.36 & 0.29 \\
\hline Other expenses $(\$)$ & 0.84 & 0.81 & 0.38 & 0.60 & 0.94 \\
\hline Total expenses $(\$)$ & 17.88 & 17.39 & 2.38 & 16.57 & 18.66 \\
\hline Net farm income from operations $(\$)$ & $(0.21)$ & 0.17 & 2.39 & 1.15 & $(0.89)$ \\
\hline Gain on sale of capital assets (\$) & 0.12 & 0.00 & 0.75 & 0.54 & $(0.06)$ \\
\hline
\end{tabular}


Table 1.2. DBAP 2002 Summary - Revenues and expenses by state and overall average, median, and standard deviation.

Net farm income $(\$)$

$(0.10)$

0.81

2.44

1.69

$(0.96)$

Table 1.3. DBAP 2002 Summary - Financial performance by state and overall average, median, and standard deviation.

\begin{tabular}{|c|c|c|c|c|c|}
\hline \multirow[b]{2}{*}{ Category } & \multicolumn{3}{|c|}{ Overall } & \multicolumn{2}{|c|}{ State Averages } \\
\hline & Average & Median & $\mathrm{Std}^{1}$ & Florida & Georgia \\
\hline Number of farms & 27 & 27 & 27 & 18 & 8 \\
\hline \multicolumn{6}{|l|}{ Liquidity } \\
\hline Current ratio & 29.30 & 0.67 & 145.39 & 97.05 & 0.76 \\
\hline Working capital & $(57,611)$ & $(17,764)$ & 917,514 & 459,761 & $(291,918)$ \\
\hline \multicolumn{6}{|l|}{ Solvency } \\
\hline Debt to asset ratio & 0.40 & 0.37 & 0.24 & 0.34 & 0.41 \\
\hline Equity to asset ratio & 0.60 & 0.63 & 0.24 & 0.66 & 0.59 \\
\hline Debt to equity ratio & 1.10 & 0.58 & 1.39 & 1.22 & 1.00 \\
\hline \multicolumn{6}{|l|}{ Profitability } \\
\hline Rate of return on assets & $(0.02)$ & $(0.00)$ & 0.08 & 0.02 & $(0.04)$ \\
\hline Rate of return on equity & $(0.08)$ & $(0.05)$ & 0.19 & $(0.03)$ & $(0.11)$ \\
\hline Operating profit margin ratio & $(0.03)$ & $(0.01)$ & 0.13 & 0.02 & $(0.06)$ \\
\hline \multicolumn{6}{|l|}{ Financial efficiency } \\
\hline Asset turnover rate & 0.69 & 0.63 & 0.38 & 0.92 & 0.61 \\
\hline Operating expense ratio & 0.88 & 0.86 & 0.15 & 0.82 & 0.92 \\
\hline Depreciation expense ratio & 0.11 & 0.10 & 0.06 & 0.11 & 0.10 \\
\hline Interest expense ratio & 0.03 & 0.02 & 0.03 & 0.02 & 0.03 \\
\hline Net farm income ratio & $(0.02)$ & 0.01 & 0.14 & 0.05 & $(0.05)$ \\
\hline \multicolumn{6}{|l|}{ Repayment capacity } \\
\hline Cash flow coverage ratio & 28.45 & 0.39 & 141.58 & 94.01 & 0.76 \\
\hline Term debt coverage ratio ${ }^{1}$ & 1.24 & 0.77 & 2.40 & 0.73 & 1.25 \\
\hline Capital replacement margin ${ }^{2}$ & $(63,901)$ & $(36,006)$ & 833,244 & 174,824 & $(181,959)$ \\
\hline
\end{tabular}

${ }^{1}$ Term debt and capital lease coverage ratio.

${ }^{2}$ Capital replacement and term debt repayment margin.

Table 1.4. DBAP 2002 Summary - Balance sheet by state and overall average, median, and standard deviation.

\begin{tabular}{|c|c|c|c|c|c|}
\hline \multirow[b]{2}{*}{ Category } & \multicolumn{3}{|c|}{ Overall } & \multicolumn{2}{|c|}{ State Averages } \\
\hline & Average & Median & Std $^{1}$ & Florida & Georgia \\
\hline Number of farms & 27 & 27 & 27 & 18 & 8 \\
\hline \multicolumn{6}{|l|}{ Balance sheet (January 1) } \\
\hline Current assets/cow (\$) & 526 & 303 & 503 & 915 & 367 \\
\hline Total assets/cow (\$) & 5,262 & 4,312 & 2,963 & 4,893 & 5,430 \\
\hline Current liabilities/cow (\$) & 442 & 309 & 368 & 445 & 438 \\
\hline Total liabilities/cow $(\$)$ & 1,705 & 1,235 & 1,189 & 1,355 & 1,762 \\
\hline Equity/cow (\$) & 3,562 & 2,375 & 2,866 & 3,538 & 3,676 \\
\hline \multicolumn{6}{|c|}{ Balance sheet (December 31) } \\
\hline Current assets/cow $(\$)$ & 486 & 287 & 440 & 783 & 367 \\
\hline Total assets/cow $(\$)$ & 5,449 & 4,191 & 3,004 & 5,014 & 5,671 \\
\hline
\end{tabular}


Table 1.4. DBAP 2002 Summary - Balance sheet by state and overall average, median, and standard deviation.

\begin{tabular}{lccccc} 
Current liabilities/cow (\$) & 517 & 466 & 514 & 276 & 640 \\
Total liabilities/cow (\$) & 1,962 & 1,791 & 1,143 & 1,530 & 2,082 \\
Equity/cow $(\$)$ & 3,487 & 2,316 & 2,899 & 3,484 & 3,589 \\
\hline
\end{tabular}

Table 2.1. DBAP 2002 Summary - Business size and production efficiency by average number of cows and milk per cow.

\begin{tabular}{|c|c|c|c|c|c|c|}
\hline \multirow[b]{2}{*}{ Category } & \multicolumn{3}{|c|}{ Average number of cows } & \multicolumn{3}{|c|}{ Milk per cow (lbs/year) } \\
\hline & $<500$ & $\begin{array}{l}500- \\
990\end{array}$ & $>990$ & $<15,000$ & $\begin{array}{l}15,000- \\
18,000\end{array}$ & $>18,000$ \\
\hline Number of farms & 9 & 9 & 9 & 9 & 9 & 9 \\
\hline \multicolumn{7}{|l|}{ Business Size } \\
\hline Average number of cows & 279 & 774 & 2,452 & 897 & 967 & 1,641 \\
\hline Average number of heifers & 183 & 259 & 1,308 & 277 & 553 & 920 \\
\hline Milk sold (million lbs) & 4.38 & 12.85 & 46.46 & 12.20 & 16.27 & 35.22 \\
\hline FTE workers & 6 & 13 & 40 & 13 & 16 & 30 \\
\hline Acres of pasture + cultivated land & 262 & 352 & 1,204 & 672 & 454 & 691 \\
\hline \multicolumn{7}{|l|}{ Production Efficiency } \\
\hline Milk sold/cow (lbs) & 15,778 & 16,444 & 18,208 & 13,030 & 16,784 & 20,615 \\
\hline Cows/FTE worker & 45 & 62 & 75 & 65 & 63 & 53 \\
\hline Milk sold/FTE worker (million lbs) & 0.69 & 1.00 & 1.33 & 0.87 & 1.06 & 1.10 \\
\hline Cull rate & 0.36 & 0.31 & 0.36 & 0.36 & 0.37 & 0.30 \\
\hline
\end{tabular}

Table 2.2. DBAP 2002 Summary - Revenues and expenses by average number of cows and milk per cow.

\begin{tabular}{|c|c|c|c|c|c|c|}
\hline \multirow{3}{*}{ Category } & \multicolumn{3}{|c|}{ Average number of cows } & \multicolumn{3}{|c|}{ Milk per cow (lbs / year) } \\
\hline & \multicolumn{3}{|c|}{$500-$} & \multicolumn{3}{|c|}{$15,000-$} \\
\hline & $<500$ & 990 & $>990$ & $<15,000$ & 18,000 & $>18,000$ \\
\hline Number of farms & 9 & 9 & 9 & 9 & 9 & 9 \\
\hline \multicolumn{7}{|l|}{ Revenues (per cwt) } \\
\hline Milk sold (\$) & 15.81 & 15.91 & 16.44 & 15.94 & 16.40 & 15.82 \\
\hline Raised, leased cow sales (\$) & 0.89 & 0.40 & 0.67 & 0.82 & 0.53 & 0.61 \\
\hline Heifer sales $(\$)$ & 0.38 & 0.03 & $(0.03)$ & $(0.13)$ & 0.33 & 0.19 \\
\hline Gain on purchased livestock sales $(\$)$ & $(0.10)$ & $(0.38)$ & $(0.49)$ & $(0.64)$ & $(0.46)$ & 0.13 \\
\hline Other revenues $(\$)$ & 2.25 & 0.61 & 0.62 & 1.56 & 0.87 & 1.05 \\
\hline Total revenues (\$) & 19.24 & 16.56 & 17.21 & 17.55 & 17.67 & 17.80 \\
\hline \multicolumn{7}{|l|}{ Expenses (per cwt) } \\
\hline Personnel (\$) & 2.97 & 2.76 & 2.91 & 2.94 & 2.71 & 2.99 \\
\hline Purchased feed $(\$)$ & 6.50 & 7.40 & 7.09 & 7.20 & 6.88 & 6.92 \\
\hline Crops (\$) & 0.51 & 0.21 & 0.29 & 0.28 & 0.30 & 0.42 \\
\hline Machinery (\$) & 0.97 & 0.59 & 0.86 & 0.72 & 0.87 & 0.84 \\
\hline Livestock (\$) & 1.95 & 1.79 & 1.79 & 1.43 & 1.60 & 2.50 \\
\hline Milk marketing $(\$)$ & 1.07 & 1.16 & 1.01 & 0.92 & 1.17 & 1.15 \\
\hline Buildings and land (\$) & 0.81 & 0.68 & 0.58 & 0.45 & 0.96 & 0.67 \\
\hline Interest (\$) & 0.69 & 0.46 & 0.38 & 0.63 & 0.57 & 0.34 \\
\hline
\end{tabular}


Table 2.2. DBAP 2002 Summary - Revenues and expenses by average number of cows and milk per cow.

\begin{tabular}{|c|c|c|c|c|c|c|}
\hline \multicolumn{7}{|l|}{ Depreciation: } \\
\hline Livestock (\$) & 1.12 & 0.86 & 1.15 & 1.33 & 1.30 & 0.50 \\
\hline Machinery $(\$)$ & 0.61 & 0.57 & 0.46 & 0.49 & 0.58 & 0.58 \\
\hline Buildings $(\$)$ & 0.38 & 0.22 & 0.30 & 0.35 & 0.21 & 0.34 \\
\hline Other expenses $(\$)$ & 1.07 & 0.72 & 0.73 & 1.10 & 0.73 & 0.70 \\
\hline Total expenses $(\$)$ & 18.65 & 17.44 & 17.56 & 17.83 & 17.88 & 17.94 \\
\hline Net farm income from operations $(\$)$ & 0.59 & $(0.88)$ & $(0.35)$ & $(0.28)$ & $(0.21)$ & $(0.14)$ \\
\hline Gain on sale of capital assets $(\$)$ & $(0.01)$ & $(0.04)$ & 0.40 & $(0.14)$ & 0.48 & 0.00 \\
\hline Net farm income $(\$)$ & 0.57 & $(0.92)$ & 0.06 & $(0.42)$ & 0.27 & $(0.14)$ \\
\hline
\end{tabular}

Table 2.3. DBAP 2002 Summary - Financial performance by average number of cows and milk per cow.

\begin{tabular}{|c|c|c|c|c|c|c|}
\hline \multirow{3}{*}{ Category } & \multicolumn{3}{|c|}{ Average number of cows } & \multicolumn{3}{|c|}{ Milk per cow (lbs/year) } \\
\hline & \multicolumn{3}{|c|}{$500-$} & \multicolumn{3}{|c|}{$15,000-$} \\
\hline & $<500$ & 990 & $>990$ & $<15,000$ & 18,000 & $>18,000$ \\
\hline Number of farms & 9 & 9 & 9 & 9 & 9 & 9 \\
\hline \multicolumn{7}{|l|}{ Liquidity } \\
\hline Current ratio & 84.50 & 1.90 & 1.51 & 0.77 & 84.99 & 2.14 \\
\hline Working capital & $(36,066)$ & $(175,900)$ & 39,132 & $(67,006)$ & 109,981 & $(215,808)$ \\
\hline \multicolumn{7}{|l|}{ Solvency } \\
\hline Debt to asset ratio & 0.45 & 0.35 & 0.40 & 0.42 & 0.44 & 0.34 \\
\hline Equity to asset ratio & 0.55 & 0.65 & 0.60 & 0.58 & 0.56 & 0.66 \\
\hline Debt to equity ratio & 1.74 & 0.76 & 0.80 & 0.89 & 1.67 & 0.74 \\
\hline \multicolumn{7}{|l|}{ Profitability } \\
\hline Rate of return on assets & $(0.01)$ & $(0.03)$ & $(0.00)$ & $(0.02)$ & $(0.02)$ & $(0.01)$ \\
\hline Rate of return on equity & $(0.14)$ & $(0.09)$ & $(0.02)$ & $(0.05)$ & $(0.15)$ & $(0.05)$ \\
\hline Operating profit margin ratio & $(0.03)$ & $(0.05)$ & $(0.01)$ & $(0.05)$ & $(0.03)$ & $(0.02)$ \\
\hline \multicolumn{7}{|l|}{ Financial efficiency } \\
\hline Asset turnover rate & 0.53 & 0.80 & 0.75 & 0.44 & 0.74 & 0.90 \\
\hline Operating expense ratio & 0.83 & 0.92 & 0.89 & 0.86 & 0.87 & 0.91 \\
\hline Depreciation expense ratio & 0.11 & 0.10 & 0.11 & 0.12 & 0.12 & 0.08 \\
\hline Interest expense ratio & 0.04 & 0.03 & 0.02 & 0.04 & 0.03 & 0.02 \\
\hline Net farm income ratio & 0.03 & $(0.06)$ & $(0.03)$ & $(0.02)$ & $(0.02)$ & $(0.01)$ \\
\hline \multicolumn{7}{|l|}{ Repayment capacity } \\
\hline Cash flow coverage ratio & 82.28 & 1.49 & 1.58 & 1.32 & 82.94 & 1.08 \\
\hline Term debt coverage ratio ${ }^{1}$ & 1.60 & 0.47 & 1.65 & 1.90 & 1.76 & 0.06 \\
\hline Capital replacement margin ${ }^{2}$ & $(5,789)$ & $(64,520)$ & $(121,394)$ & 41,017 & 54,750 & $(287,470)$ \\
\hline
\end{tabular}

\footnotetext{
${ }^{1}$ Term debt and capital lease coverage ratio.

${ }^{2}$ Capital replacement and term debt repayment margin.
} 
Table 2.4. DBAP 2002 Summary - Balance sheet by average number of cows and milk per cow.

\begin{tabular}{|c|c|c|c|c|c|c|}
\hline \multirow[b]{2}{*}{ Category } & \multicolumn{3}{|c|}{ Average number of cows } & \multicolumn{3}{|c|}{ Milk per cow (Ibs/year) } \\
\hline & \multicolumn{3}{|c|}{$500-$} & \multicolumn{3}{|c|}{$15,000-$} \\
\hline Number of farms & 9 & 9 & 9 & 9 & 9 & 9 \\
\hline \multicolumn{7}{|l|}{ Balance sheet (January 1) } \\
\hline Current assets/cow (\$) & 541 & 342 & 696 & 186 & 569 & 824 \\
\hline Total assets/cow (\$) & 6,968 & 4,801 & 4,016 & 6,514 & 4,004 & 5,267 \\
\hline Current liabilities/cow (\$) & 441 & 387 & 498 & 397 & 398 & 530 \\
\hline Total liabilities/cow (\$) & 2,402 & 1,438 & 1,274 & 2,202 & 1,486 & 1,426 \\
\hline Equity/cow (\$) & 4,566 & 3,375 & 2,745 & 4,312 & 2,533 & 3,841 \\
\hline \multicolumn{7}{|c|}{ Balance sheet (December 31) } \\
\hline Current assets/cow (\$) & 475 & 313 & 672 & 222 & 481 & 756 \\
\hline Total assets/cow (\$) & 6,890 & 5,061 & 4,395 & 6,747 & 4,342 & 5,257 \\
\hline Current liabilities/cow (\$) & 408 & 534 & 609 & 359 & 489 & 703 \\
\hline Total liabilities/cow (\$) & 2,422 & 1,713 & 1,751 & 2,362 & 1,841 & 1,683 \\
\hline Equity/cow (\$) & 4,468 & 3,348 & 2,645 & 4,385 & 2,501 & 3,574 \\
\hline
\end{tabular}

Table 3.1. DBAP 2002 Summary - Business size and production efficiency by net farm income per cwt and rate of return on assets.

\begin{tabular}{|c|c|c|c|c|c|c|}
\hline \multirow[b]{2}{*}{ Category } & \multicolumn{3}{|c|}{ Net farm income/cwt $(\$)$} & \multicolumn{3}{|c|}{ Rate of return on assets (\%) } \\
\hline & \multicolumn{3}{|c|}{$\$(1.00)-$} & \multicolumn{3}{|c|}{$(3.1) \%-$} \\
\hline Number of farms & 9 & 9 & 9 & 9 & 9 & 9 \\
\hline \multicolumn{7}{|l|}{ Business size } \\
\hline Average number of cows & 1,010 & 1,730 & 765 & 1,165 & 678 & 1,662 \\
\hline Average number of heifers & 609 & 922 & 219 & 731 & 202 & 817 \\
\hline Milk sold (million lbs) & 18.70 & 30.38 & 14.61 & 21.72 & 10.19 & 31.79 \\
\hline FTE workers & 21 & 28 & 10 & 23 & 12 & 24 \\
\hline Acres of pasture + cultivated land & 462 & 939 & 415 & 441 & 542 & 834 \\
\hline \multicolumn{7}{|l|}{ Production efficiency } \\
\hline Milk sold/cow (lbs) & 16,451 & 16,661 & 17,318 & 16,560 & 16,104 & 17,766 \\
\hline Cows/FTE worker & 58 & 63 & 60 & 57 & 52 & 73 \\
\hline Milk sold/FTE worker (million lbs) & 0.94 & 1.03 & 1.06 & 0.93 & 0.82 & 1.28 \\
\hline Cull rate & 0.31 & 0.39 & 0.33 & 0.35 & 0.33 & 0.35 \\
\hline
\end{tabular}

Table 3.2. DBAP 2002 Summary - Revenues and expenses by net farm income per cwt and rate of return on assets.

\begin{tabular}{|c|c|c|c|c|c|c|}
\hline \multirow{3}{*}{ Category } & \multicolumn{3}{|c|}{ Net farm income / cwt (\$) } & \multicolumn{3}{|c|}{ Rate of return on assets (\%) } \\
\hline & \multicolumn{3}{|c|}{$\$(1.00)-$} & \multicolumn{3}{|c|}{$(3.1) \%-$} \\
\hline & $<\$(1.00)$ & $\$ 1.10$ & $>\$ 1.10$ & $<(3.1) \%$ & $3.0 \%$ & $>3.0 \%$ \\
\hline Number of farms & 9 & 9 & 9 & 9 & 9 & 9 \\
\hline \multicolumn{7}{|c|}{ Revenues (per cwt) } \\
\hline Milk sold (\$) & 16.20 & 15.89 & 16.07 & 16.34 & 15.93 & 15.90 \\
\hline
\end{tabular}


Table 3.2. DBAP 2002 Summary - Revenues and expenses by net farm income per cwt and rate of return on assets.

\begin{tabular}{|c|c|c|c|c|c|c|}
\hline Raised, leased cow sales $(\$)$ & 0.48 & 0.95 & 0.54 & 0.81 & 0.80 & 0.35 \\
\hline Heifer sales $(\$)$ & 0.15 & 0.04 & 0.19 & $(0.00)$ & 0.15 & 0.24 \\
\hline Gain on purchased livestock sales (\$) & $(0.32)$ & $(0.87)$ & 0.22 & $(1.11)$ & $(0.13)$ & 0.27 \\
\hline Other revenues $(\$)$ & 0.76 & 1.31 & 1.41 & 0.89 & 1.86 & 0.73 \\
\hline Total revenues $(\$)$ & 17.27 & 17.32 & 18.43 & 16.92 & 18.61 & 17.48 \\
\hline \multicolumn{7}{|l|}{ Expenses (per cwt) } \\
\hline Personnel (\$) & 3.13 & 3.15 & 2.37 & 3.12 & 3.14 & 2.38 \\
\hline Purchased feed $(\$)$ & 8.87 & 6.51 & 5.62 & 8.93 & 6.17 & 5.89 \\
\hline Crops (\$) & 0.23 & 0.18 & 0.60 & 0.13 & 0.50 & 0.38 \\
\hline Machinery (\$) & 0.87 & 0.86 & 0.69 & 0.86 & 0.76 & 0.81 \\
\hline Livestock (\$) & 2.12 & 1.71 & 1.69 & 2.04 & 1.71 & 1.78 \\
\hline Milk marketing $(\$)$ & 0.92 & 0.96 & 1.36 & 0.99 & 1.16 & 1.09 \\
\hline Buildings and land (\$) & 0.63 & 0.49 & 0.95 & 0.61 & 0.95 & 0.51 \\
\hline Interest $(\$)$ & 0.61 & 0.52 & 0.41 & 0.46 & 0.66 & 0.42 \\
\hline \multicolumn{7}{|l|}{ Depreciation: } \\
\hline Livestock (\$) & 0.79 & 1.20 & 1.14 & 0.84 & 1.05 & 1.24 \\
\hline Machinery $(\$)$ & 0.55 & 0.44 & 0.65 & 0.43 & 0.74 & 0.46 \\
\hline Buildings (\$) & 0.29 & 0.36 & 0.25 & 0.33 & 0.36 & 0.20 \\
\hline Other expenses $(\$)$ & 1.00 & 0.86 & 0.67 & 0.83 & 1.01 & 0.69 \\
\hline Total expenses (\$) & 20.01 & 17.25 & 16.39 & 19.59 & 18.22 & 15.84 \\
\hline Net farm income from operations $(\$)$ & $(2.74)$ & 0.07 & 2.04 & $(2.66)$ & 0.39 & 1.64 \\
\hline Gain on sale of capital assets $(\$)$ & $(0.21)$ & 0.41 & 0.14 & 0.12 & 0.23 & $(0.01)$ \\
\hline Net farm income $(\$)$ & $(2.95)$ & 0.48 & 2.18 & $(2.54)$ & 0.62 & 1.63 \\
\hline
\end{tabular}


Table 3.3. DBAP 2002 Summary - Financial performance by net farm income per cwt and rate of return on assets.

\begin{tabular}{|c|c|c|c|c|c|c|}
\hline \multirow[b]{2}{*}{ Category } & \multicolumn{3}{|c|}{ Net farm income/cwt $(\$)$} & \multicolumn{3}{|c|}{ Rate of return on assets (\%) } \\
\hline & $<\$(1.00)$ & $\begin{array}{c}\$(1.00)- \\
\$ 1.10\end{array}$ & $>\$ 1.10$ & $<(3.1) \%$ & $\begin{array}{c}(3.1) \%- \\
3.0 \%\end{array}$ & $>3.0 \%$ \\
\hline Number of farms & 9 & 9 & 9 & 9 & 9 & 9 \\
\hline \multicolumn{7}{|l|}{ Liquidity } \\
\hline Current ratio & 0.40 & 0.96 & 86.54 & 0.46 & 0.68 & 86.76 \\
\hline Working capital & $(328,430)$ & $(330,082)$ & 485,678 & $(276,793)$ & $(52,919)$ & 156,878 \\
\hline \multicolumn{7}{|l|}{ Solvency } \\
\hline Debt to asset ratio & 0.46 & 0.35 & 0.38 & 0.46 & 0.41 & 0.33 \\
\hline Equity to asset ratio & 0.54 & 0.65 & 0.62 & 0.54 & 0.59 & 0.67 \\
\hline Debt to equity ratio & 1.23 & 0.84 & 1.23 & 1.26 & 1.33 & 0.72 \\
\hline \multicolumn{7}{|l|}{ Profitability } \\
\hline Rate of return on assets & $(0.10)$ & 0.01 & 0.04 & $(0.11)$ & $(0.01)$ & 0.07 \\
\hline Rate of return on equity & $(0.25)$ & $(0.02)$ & 0.02 & $(0.26)$ & $(0.07)$ & 0.09 \\
\hline Operating profit margin ratio & $(0.15)$ & 0.00 & 0.06 & $(0.19)$ & $(0.00)$ & 0.09 \\
\hline \multicolumn{7}{|l|}{ Financial efficiency } \\
\hline Asset turnover rate & 0.69 & 0.73 & 0.66 & 0.64 & 0.58 & 0.86 \\
\hline Operating expense ratio & 1.03 & 0.86 & 0.75 & 1.04 & 0.83 & 0.77 \\
\hline Depreciation expense ratio & 0.10 & 0.11 & 0.11 & 0.10 & 0.11 & 0.11 \\
\hline Interest expense ratio & 0.03 & 0.03 & 0.02 & 0.03 & 0.04 & 0.02 \\
\hline Net farm income ratio & $(0.16)$ & $(0.01)$ & 0.11 & $(0.17)$ & 0.02 & 0.09 \\
\hline \multicolumn{7}{|l|}{ Repayment capacity } \\
\hline Cash flow coverage ratio & $(0.05)$ & 1.45 & 83.95 & 0.13 & 1.03 & 84.19 \\
\hline Term debt coverage ratio $^{1}$ & $(0.35)$ & 1.83 & 2.24 & $(0.40)$ & 1.49 & 2.62 \\
\hline Capital replacement margin ${ }^{2}$ & $(366,031)$ & $(244,367)$ & 418,696 & $(463,056)$ & 88,335 & 183,019 \\
\hline
\end{tabular}

${ }^{1}$ Term debt and capital lease coverage ratio.

${ }^{2}$ Capital replacement and term debt repayment margin.

Table 3.4. DBAP 2002 Summary - Balance sheet by net farm income per cwt and rate of return on assets.

\begin{tabular}{|c|c|c|c|c|c|c|}
\hline \multirow[b]{2}{*}{ Category } & \multicolumn{3}{|c|}{ Net farm income/cwt $(\$)$} & \multicolumn{3}{|c|}{ Rate of return on assets (\%) } \\
\hline & $<\$(1.00)$ & $\begin{array}{c}\$(1.00)- \\
\$ 1.10 \\
\end{array}$ & $>\$ 1.10$ & $<(3.1) \%$ & $\begin{array}{c}(3.1) \%- \\
3.0 \% \\
\end{array}$ & $>3.0 \%$ \\
\hline Number of farms & 9 & 9 & 9 & 9 & 9 & 9 \\
\hline \multicolumn{7}{|l|}{ Balance sheet (January 1) } \\
\hline Current assets/cow (\$) & 289 & 406 & 884 & 291 & 487 & 802 \\
\hline Total assets/cow $(\$)$ & 4,285 & 5,392 & 6,109 & 5,007 & 6,632 & 4,146 \\
\hline Current liabilities/cow (\$) & 338 & 486 & 502 & 321 & 493 & 512 \\
\hline Total liabilities/cow (\$) & 1,419 & 1,631 & 2,064 & 1,553 & 2,089 & 1,472 \\
\hline Equity/cow (\$) & 2,878 & 3,764 & 4,045 & 3,466 & 4,544 & 2,678 \\
\hline \multicolumn{7}{|l|}{ Balance sheet (December 31) } \\
\hline Current assets/cow $(\$)$ & 255 & 457 & 747 & 296 & 430 & 733 \\
\hline Total assets/cow $(\$)$ & 4,316 & 5,954 & 6,076 & 5,299 & 6,804 & 4,243 \\
\hline Current liabilities/cow $(\$)$ & 680 & 586 & 286 & 666 & 453 & 431 \\
\hline Total liabilities/cow $(\$)$ & 1,859 & 2,024 & 2,002 & 2,214 & 2,159 & 1,513 \\
\hline Equity/cow (\$) & 2,456 & 3,930 & 4,074 & 3,085 & 4,645 & 2,730 \\
\hline
\end{tabular}


Table 4.1. DBAP 2002 Summary - Business size and production efficiency by assets per cow and liabilities per cow.

\begin{tabular}{|c|c|c|c|c|c|c|}
\hline \multirow[b]{3}{*}{ Category } & \multicolumn{3}{|c|}{ Assets per cow (\$) } & \multicolumn{3}{|c|}{ Liabilities per cow $(\$)$} \\
\hline & \multicolumn{3}{|c|}{$\$ 3,900-$} & \multicolumn{3}{|c|}{$\$ 1,070-$} \\
\hline & $<\$ 3,900$ & $\$ 5,000$ & $>\$ 5,000$ & $<\$ 1,070$ & $\$ 2,100$ & $>\$ 2,100$ \\
\hline Number of farms & 9 & 9 & 9 & 9 & 9 & 9 \\
\hline \multicolumn{7}{|l|}{ Business size } \\
\hline Average number of cows & 1,541 & 1,009 & 955 & 1,038 & 1,710 & 757 \\
\hline Average number of heifers & 738 & 418 & 594 & 619 & 939 & 192 \\
\hline Milk sold (million lbs) & 27.17 & 17.70 & 18.83 & 20.01 & 29.89 & 13.79 \\
\hline FTE workers & 28 & 15 & 17 & 23 & 26 & 10 \\
\hline Acres of pasture + cultivated land & 607 & 593 & 617 & 510 & 849 & 458 \\
\hline \multicolumn{7}{|l|}{ Production efficiency } \\
\hline Milk sold/cow (lbs) & 17,113 & 15,925 & 17,392 & 17,823 & 16,161 & 16,446 \\
\hline Cows/FTE worker & 66 & 66 & 50 & 48 & 69 & 64 \\
\hline Milk sold/FTE worker (million lbs) & 1.08 & 1.07 & 0.87 & 0.85 & 1.11 & 1.07 \\
\hline Cull rate & 0.35 & 0.33 & 0.35 & 0.36 & 0.31 & 0.35 \\
\hline
\end{tabular}

Table 4.2. DBAP 2002 Summary - Revenues and expenses by assets per cow and liabilities per cow.

\begin{tabular}{|c|c|c|c|c|c|c|}
\hline \multirow[b]{2}{*}{ Category } & \multicolumn{3}{|c|}{ Assets per cow $(\$)$} & \multicolumn{3}{|c|}{ Liabilities per cow $(\$)$} \\
\hline & \multicolumn{3}{|c|}{$\$ 3,900-$} & \multicolumn{2}{|r|}{$\$ 1,070$} & $>\$ 2,100$ \\
\hline Number of farms & 9 & 9 & 9 & 9 & 9 & 9 \\
\hline \multicolumn{7}{|l|}{ Revenues (per cwt) } \\
\hline Milk sold (\$) & 16.14 & 16.20 & 15.82 & 15.68 & 16.45 & 16.03 \\
\hline Raised, leased cow sales $(\$)$ & 0.73 & 0.45 & 0.78 & 0.63 & 0.69 & 0.64 \\
\hline Heifer sales $(\$)$ & $(0.19)$ & 0.38 & 0.19 & 0.06 & $(0.19)$ & 0.51 \\
\hline Gain on purchased livestock sales (\$) & $(0.67)$ & $(0.09)$ & $(0.22)$ & 0.13 & $(1.07)$ & $(0.03)$ \\
\hline Other revenues $(\$)$ & 0.52 & 0.84 & 2.12 & 1.92 & 0.58 & 0.98 \\
\hline Total revenues $(\$)$ & 16.54 & 17.77 & 18.70 & 18.42 & 16.46 & 18.13 \\
\hline \multicolumn{7}{|l|}{ Expenses (per cwt) } \\
\hline Personnel (\$) & 2.68 & 2.41 & 3.55 & 2.89 & 2.78 & 2.97 \\
\hline Purchased feed $(\$)$ & 7.22 & 6.37 & 7.40 & 6.78 & 7.68 & 6.53 \\
\hline Crops $(\$)$ & 0.14 & 0.49 & 0.38 & 0.38 & 0.30 & 0.33 \\
\hline Machinery (\$) & 0.83 & 0.66 & 0.94 & 0.95 & 0.71 & 0.76 \\
\hline Livestock (\$) & 1.97 & 1.58 & 1.98 & 2.04 & 1.76 & 1.73 \\
\hline Milk marketing (\$) & 1.15 & 1.05 & 1.04 & 1.25 & 0.97 & 1.01 \\
\hline Buildings and land (\$) & 0.66 & 0.89 & 0.53 & 0.51 & 0.81 & 0.75 \\
\hline Interest (\$) & 0.45 & 0.59 & 0.49 & 0.31 & 0.50 & 0.72 \\
\hline \multicolumn{7}{|l|}{ Depreciation: } \\
\hline Livestock (\$) & 0.98 & 1.44 & 0.72 & 0.79 & 1.01 & 1.33 \\
\hline Machinery (\$) & 0.72 & 0.45 & 0.48 & 0.74 & 0.48 & 0.41 \\
\hline Buildings (\$) & 0.26 & 0.25 & 0.39 & 0.37 & 0.30 & 0.23 \\
\hline Other expenses $(\$)$ & 0.84 & 0.84 & 0.85 & 0.79 & 0.87 & 0.87 \\
\hline Total expenses $(\$)$ & 17.89 & 17.02 & 18.74 & 17.81 & 18.19 & 17.65 \\
\hline Net farm income from operations $(\$)$ & $(1.35)$ & 0.76 & $(0.04)$ & 0.61 & $(1.72)$ & 0.48 \\
\hline Gain on sale of capital assets (\$) & 0.36 & $(0.03)$ & 0.01 & 0.17 & 0.20 & $(0.02)$ \\
\hline
\end{tabular}


Table 4.3. DBAP 2002 Summary - Financial performance by assets per cow and liabilities per cow.

\begin{tabular}{|c|c|c|c|c|c|c|}
\hline \multirow[b]{2}{*}{ Category } & \multicolumn{3}{|c|}{ Assets per cow $(\$)$} & \multicolumn{3}{|c|}{ Liabilities per cow $(\$)$} \\
\hline & $<\$ 3,900$ & $\begin{array}{l}\$ 3,900- \\
\$ 5,000\end{array}$ & $>\$ 5,000$ & $<\$ 1,070$ & $\begin{array}{l}\$ 1,070- \\
\$ 2,100\end{array}$ & $>\$ 2,100$ \\
\hline Number of farms & 9 & 9 & 9 & 9 & 9 & 9 \\
\hline \multicolumn{7}{|l|}{ Liquidity } \\
\hline Current ratio & 0.74 & 85.28 & 1.87 & 86.22 & 0.86 & 0.83 \\
\hline Working capital & $(126,185)$ & 404,342 & $(450,990)$ & 110,984 & $(446,171)$ & 162,354 \\
\hline \multicolumn{7}{|l|}{ Solvency } \\
\hline Debt to asset ratio & 0.48 & 0.44 & 0.28 & 0.16 & 0.45 & 0.58 \\
\hline Equity to asset ratio & 0.52 & 0.56 & 0.72 & 0.84 & 0.55 & 0.42 \\
\hline Debt to equity ratio & 1.40 & 1.38 & 0.53 & 0.21 & 0.93 & 2.16 \\
\hline \multicolumn{7}{|l|}{ Profitability } \\
\hline Rate of return on assets & $(0.06)$ & 0.03 & $(0.02)$ & 0.00 & $(0.06)$ & 0.01 \\
\hline Rate of return on equity & $(0.19)$ & $(0.02)$ & $(0.04)$ & $(0.01)$ & $(0.14)$ & $(0.09)$ \\
\hline Operating profit margin ratio & $(0.08)$ & 0.03 & $(0.04)$ & 0.00 & $(0.10)$ & 0.00 \\
\hline \multicolumn{7}{|l|}{ Financial efficiency } \\
\hline Asset turnover rate & 0.97 & 0.66 & 0.45 & 0.84 & 0.63 & 0.60 \\
\hline Operating expense ratio & 0.94 & 0.80 & 0.90 & 0.85 & 0.97 & 0.82 \\
\hline Depreciation expense ratio & 0.12 & 0.12 & 0.08 & 0.10 & 0.11 & 0.11 \\
\hline Interest expense ratio & 0.03 & 0.03 & 0.03 & 0.02 & 0.03 & 0.04 \\
\hline Net farm income ratio & $(0.09)$ & 0.04 & $(0.00)$ & 0.03 & $(0.11)$ & 0.03 \\
\hline \multicolumn{7}{|l|}{ Repayment capacity } \\
\hline Cash flow coverage ratio & 0.98 & 83.38 & 1.00 & 83.42 & 0.84 & 1.09 \\
\hline Term debt coverage ratio $^{1}$ & 0.51 & 2.59 & 0.63 & 0.98 & 0.50 & 2.24 \\
\hline Capital replacement margin $^{2}$ & $(247,029)$ & 441,946 & $(386,619)$ & $(13,234)$ & $(380,602)$ & 202,133 \\
\hline
\end{tabular}

${ }^{1}$ Term debt and capital lease coverage ratio.

${ }^{2}$ Capital replacement and term debt repayment margin.

Table 4.4. DBAP 2002 Summary - Balance sheet by assets per cow and liabilities per cow.

\begin{tabular}{|c|c|c|c|c|c|c|}
\hline \multirow[b]{3}{*}{ Category } & \multicolumn{3}{|c|}{ Assets per cow $(\$)$} & \multicolumn{3}{|c|}{ Liabilities per cow $(\$)$} \\
\hline & \multicolumn{3}{|c|}{$\$ 3,900-$} & \multicolumn{3}{|c|}{$\$ 1,070-$} \\
\hline & $<\$ 3,900$ & $\$ 5,000$ & $>\$ 5,000$ & $<\$ 1,070$ & $\$ 2,100$ & $>\$ 2,100$ \\
\hline Number of farms & 9 & 9 & 9 & 9 & 9 & 9 \\
\hline \multicolumn{7}{|l|}{ Balance sheet (January 1) } \\
\hline Current assets/cow (\$) & 289 & 699 & 591 & 630 & 498 & 452 \\
\hline Total assets/cow (\$) & 3,260 & 4,230 & 8,296 & 5,715 & 3,989 & 6,081 \\
\hline Current liabilities/cow (\$) & 459 & 396 & 471 & 300 & 456 & 570 \\
\hline Total liabilities/cow (\$) & 1,396 & 1,831 & 1,887 & 699 & 1,304 & 3,111 \\
\hline Equity/cow (\$) & 1,864 & 2,402 & 6,421 & 5,032 & 2,685 & 2,970 \\
\hline \multicolumn{7}{|c|}{ Balance sheet (December 31) } \\
\hline Current assets/cow (\$) & 324 & 538 & 597 & 617 & 487 & 355 \\
\hline Total assets/cow (\$) & 3,479 & 4,295 & 8,572 & 5,624 & 4,333 & 6,389 \\
\hline Current liabilities/cow $(\$)$ & 520 & 358 & 673 & 326 & 754 & 471 \\
\hline Total liabilities/cow (\$) & 1,827 & 1,876 & 2,182 & 791 & 1,918 & 3,177 \\
\hline Equity/cow (\$) & 1,652 & 2,419 & 6,390 & 4,833 & 2,415 & 3,213 \\
\hline
\end{tabular}

